\title{
ros

\section{PROMOVENDO EDUCAÇÃO EM SAÚDE NO ESPAÇO NÃO FORMAL DE APRENDIZAGEM}

\section{Promoting health education in a non-formal learning space \\ Promoción de la educación en salud en el espacio informal de aprendizaje}

\author{
Cintia Soares Guerin \\ Universidade Regional Integrada do Alto Uruguai e das Missões - URI - Santiago (RS) - Brasil \\ Cadidja Coutinho \\ Universidade Regional Integrada do Alto Uruguai e das Missões - URI - Santiago (RS) - Brasil
}

Felippe Martins Damaceno

Universidade Estadual do Oeste do Paraná - Unioeste - Cascavel (PR) - Brasil

Nandra Martins Soares

Universidade Estadual do Centro Oeste - Unicentro - Irati (PR) - Brasil

\section{Jiam Pires Frigo}

Universidade Federal da Integração Latino-Americana - Unila - Foz do Iguaçu (PR) - Brasil

Lizandra Martins Soares

Instituto Federal de Educação, Ciência e Tecnologia Farroupilha - IF - São Vicente (RS) - Brasil

\section{RESUMO}

Objetivo: Investigar as concepções de residentes de lar de adoção sobre estilo de vida e viabilizar a promoção da saúde nesse espaço por meio de oficinas pedagógicas teórico-práticas. Métodos: Estudo realizado de setembro a novembro de 2015 em Santiago, Rio Grande do Sul, com 09 residentes femininas de um lar de adoção e dividido em três módulos: (i) contato com os responsáveis pelo lar de adoção; (ii) realização de uma avaliação prévia das residentes sobre estilo de vida por meio da ferramenta Pentáculo do bem-estar (PBE); (iii) elaboração e execução de oficinas teórico-práticas com as temáticas: higiene corporal, higiene bucal, educação sexual, alimentação saudável e atividade física. Resultados: Em relação à higiene corporal, todas as residentes apresentam o hábito de higienizar as mãos antes das refeições. Já na higiene bucal, mais da metade não têm o hábito de usar o fio dental diariamente, bem como, uma minoria se consulta periodicamente com o dentista. No que diz respeito ao tema educação sexual, todas as residentes conhecem ou já mantiveram contato com algum método contraceptivo. No quesito alimentação saudável, a maioria costuma consumir doces e raramente ingerem refrigerantes. Menos da metade das participantes costumam incluir a prática de atividade física no seu lazer. A partir da análise dos PBE, foram elaboradas as oficinas pedagógicas teórico-práticas. Conclusão: Observou-se que as participantes apresentaram uma percepção positiva sobre o estilo de vida de acordo com os parâmetros do instrumento utilizado.

Descritores: Promoção da Saúde; Saúde Coletiva; Qualidade de Vida.

\section{ABSTRACT}

Objective: To investigate foster institution residents' conceptions of lifestyle and enable the promotion of health in this space through theory and practical pedagogical workshops. Methods: A study carried out from September to November 2015 in Santiago, Rio Grande do Sul, with 09 female residents of a foster institution. The present study was divided into three modules: (i) contacting those responsible for the foster institution; (ii) carrying out a preliminary evaluation by the residents on the topic of lifestyle using as tool the Pentacle of Well-being (PWB); (iii) elaboration and execution of theory-practical workshops addressing the themes: body hygiene, oral hygiene, sex education, healthy eating and physical activity. Results: With regard to body hygiene, all residents have the habit of washing their hands before meals. As for oral hygiene, more than half do not have the habit of flossing daily and only a minority undergoes periodical consultations with the dentist. On the topic of sex education, all residents know or have had contact with some contraceptive method. Regarding healthy eating, a majority is used to ingesting lots of sweets and rarely consume soft drinks. Less than half of the participants are used to incorporating the physical activity 
practice into their leisure experiences. From the PWE analysis, theory-practical pedagogical workshops were elaborated. Conclusion: It was observed that the participants presented a positive perception of the lifestyle according to the parameters of the instrument used.

Descriptors: Health Promotion; Collective Health; Quality of Life.

\section{RESUMEN}

Objetivo: Investigar las concepciones de residentes de un hogar para adopción sobre el estilo de vida y viabilizar la promoción de la salud en ese espacio a través de talleres de pedagogía teórico/prácticos. Métodos: Estudio realizado entre septiembre y noviembre de 2015 en Santiago, Rio Grande do Sul, con 09 residentes mujeres de un hogar para adopción. El presente estudio se dividió en tres módulos: (i) el contacto con los responsables del hogar para adopción; (ii) la realización de una evaluación previa de las residentes sobre el estilo de vida a través de la herramienta Pentáculo del bienestar (PBE); (iii) la elaboración y ejecución de talleres teórico/prácticos con los temas: higiene corporal, higiene bucal, educación sexual, alimentación saludable y actividad fisica. Resultados: Respecto la higiene corporal todas las residentes tenían el hábito de higienizar las manos antes de las comidas. Sobre la higiene bucal más de la mitad de ellas no tienen el hábito de usar hilo dental a diario así como una pequeña parte va a la consulta periódica con el dentista. Respecto la educación sexual todas las residentes conocen o ya han usado algún método anticonceptivo. En el ítem alimentación saludable la gran mayoría come muchos dulces y raramente toma refrescos. Menos de la mitad de las participantes incluyen la práctica de actividad fisica en su tiempo de ocio. Se elaboraron los talleres de pedagogía teórico/práctico a partir de los análisis de los PBE. Conclusión: Se observó que las participantes presentaron una percepción positiva del estilo de vida según los parámetros del instrumento utilizado.

Descriptores: Promoción de la Salud; Salud Pública; Calidad de Vida.

\section{INTRODUÇÃO}

Durante as últimas décadas, a temática da promoção de saúde ganhou destaque em diversos âmbitos da sociedade. A promoção de saúde, ou mesmo saúde coletiva, está intimamente ligada a um conjunto de valores, como solidariedade, democracia, desenvolvimento, participação, parceria e qualidade de vida, entre outros. Trabalha ainda com a ideia de responsabilização múltipla, ou seja, com uma combinação de estratégias que vinculam ações do Estado, da comunidade, dos sistemas de saúde e, sobretudo, ações individuais ${ }^{(1-3)}$.

Ações como essas passaram a ser vistas como uma estratégia esperançosa para encarar os diversos problemas de saúde que assolam as populações humanas e o seu entorno. A promoção de saúde consiste em realizar atividades de transformação de comportamento dos indivíduos, estando relacionada a todas as práticas que possam vir a proporcionar uma melhoria na qualidade de vida da população, não se restringindo apenas a resolver problemas de doença, e sim visando aumentar a saúde e o bem-estar como um todo ${ }^{(4)}$.

Os campos de ação da promoção da saúde estão atrelados ao desenvolvimento de atitudes e habilidades pessoais que favoreçam a saúde. Dessa forma, é imprescindível a divulgação de informações sobre a educação para a saúde em todos os ambientes da sociedade uma vez que essas ações podem ser concretizadas em diversos espaços e instituições sociais ${ }^{(5)}$.

Nesse sentido, faz-se necessário desenvolver ações voltadas à promoção da educação direcionada às crianças e aos adolescentes, visto que é na fase da adolescência que ocorrem as principais mudanças físicas e psicológicas, sendo, portanto, um período de profunda aprendizagem e amadurecimento ${ }^{(6)}$.

Existe referência à educação para a saúde em escolas, já que esse é um amplo espaço de convívio social, favorecendo as relações interpessoais, além de ser um ambiente onde as crianças e os adolescentes passam maior parte de sua vida ${ }^{(7,8)}$. Entretanto, existem outras instituições que ainda necessitam do olhar da sociedade e são pouco investigadas nas pesquisas. Assim como nas escolas, crianças e adolescentes permanecem grande parte de seu tempo nos lares de adoção.

Instituições como os lares de adoção representam um espaço promissor para realizar atividades voltadas à educação científica, principalmente no tocante à promoção de saúde. Segundo o Cadastro Nacional de Crianças e Adolescentes Acolhidos (2016), o Brasil possui atualmente 45 mil crianças e adolescentes vivendo em abrigos. Segundo os dados, somente o estado do Rio Grande do Sul possui aproximadamente 5 mil crianças abrigadas ${ }^{(9)}$.

Os lares de adoção, também chamados de abrigos, são instituições destinadas a acolher e proteger crianças e adolescentes em situação de vulnerabilidade até que sua situação seja resolvida pela ação judicial. Porém, essa não é a realidade que se encontra no Brasil, pois muitos desses jovens não têm sua situação resolvida, ou não são adotados por outras famílias, e passam a ter o abrigo como um lar definitivo até a maioridade ${ }^{(10)}$.

Diante disso, assegurar o direito à saúde aos jovens que vivem em abrigos é certamente um grande desafio para os trabalhadores dessas instituições. A participação de instituições de ensino no objetivo de levar instruções de saúde aos abrigos é de fundamental importância para subsidiar melhorias na qualidade de vida dos internos, principalmente quando se considera a saúde como um direito social ${ }^{(11)}$. 
Dessa forma, o presente estudo se propôs a investigar as concepções de residentes de lares de adoção sobre estilo de vida e viabilizar a promoção da saúde nesse espaço por meio de oficinas pedagógicas teórico-práticas.

\section{MÉTODOS}

Para o desenvolvimento deste estudo utilizou-se a abordagem quantitativa e o mesmo ocorreu no período de setembro a novembro de 2015, na cidade de Santiago, Rio Grande do Sul (RS), Brasil.

Os dados foram coletados em um lar de adoção, sendo convidadas a participar do estudo crianças e adolescentes alfabetizadas, com idade compreendida entre 06 e 17 anos, todas do sexo feminino, sem deficiência física e com autorização de seus responsáveis legais. No período da coleta de dados, o lar contava com 11 residentes, das quais 9 participaram do estudo.

Realizou-se o presente estudo em três etapas: (i) contato com os responsáveis pelo lar de adoção; (ii) realização de uma avaliação prévia das residentes sobre estilo de vida por meio da ferramenta Pentáculo do bem-estar (PBE); (iii) elaboração e execução de oficinas teórico-práticas com as temáticas: higiene corporal, higiene bucal, educação sexual, alimentação saudável e atividade física.

A primeira etapa foi realizada utilizando informações fornecidas pela Prefeitura Municipal de Santiago, Rio Grande do Sul, e por sua Secretaria do Desenvolvimento Social. A segunda etapa visou identificar as principais concepções das residentes do lar de adoção sobre a promoção de saúde. Para isso, foi utilizada a ferramenta pedagógica Pentáculo do bem-estar (PBE). Essa é uma metodologia de avaliação do estilo de vida, que pode ser utilizada como base para intervenções individuais ou grupais, objetivando mudanças comportamentais e na promoção da saúde ${ }^{(12,13)}$.

A ferramenta consiste em um questionário com 15 questões objetivas de autoavaliação (quadro I) e um PBE para colorir (figura 1). O tempo médio de aplicação é entre 10 e 20 minutos, sendo constituído por 5 dimensões: higiene corporal, higiene bucal, educação sexual, alimentação saudável e atividade física. Cada uma das dimensões contém 3 questões, que podem ser respondidas a partir de uma escala tipo Likert de quatro pontos, na qual a participante afirma que: $1=$ nada/nunca, $2=$ pouco/ raramente, 3 = moderadamente/ algumas vezes e $4=$ totalmente/sempre.

Quadro I - Questionário aplicado no estudo. Santiago, RS, 2015.

\section{Questionário}

Eixo pertinente à higiene corporal

a) Você toma banho diariamente?

b) Você mantém as unhas limpas e cortadas?

c) Você lava as mãos antes de fazer as refeições?

\section{Eixo pertinente à higiene bucal}

d) Você escova os dentes após cada refeição?

e) Você faz uso do fio dental?

f) Você frequenta o dentista?

\section{Eixo pertinente à educação sexual}

g) Você conhece de algum método contraceptivo para prevenir a gravidez?

h) Você já ouviu falar em DST?

i) Você conhece o método contraceptivo chamado preservativo?

\section{Eixo pertinente à alimentação saudável}

j) Com que frequência você costuma comer doces (balas, chicles, chocolates...)?

k) Você costuma consumir refrigerante com que frequência?

l) Você costuma ter uma alimentação variada (proteínas, carboidratos, gorduras, fibras, sais minerais)?

\section{Eixo pertinente às atividades físicas}

m) Seu lazer inclui a prática de atividades físicas?

n) Ao menos duas vezes por semana você realiza exercícios que envolvam força e alongamento muscular?

o) Você caminha ou pedala como meio de deslocamento e, preferencialmente, usa as escadas ao invés do elevador?

DST: Doenças Sexualmente Transmissíveis. Fonte: Adaptado ${ }^{(12)}$ 


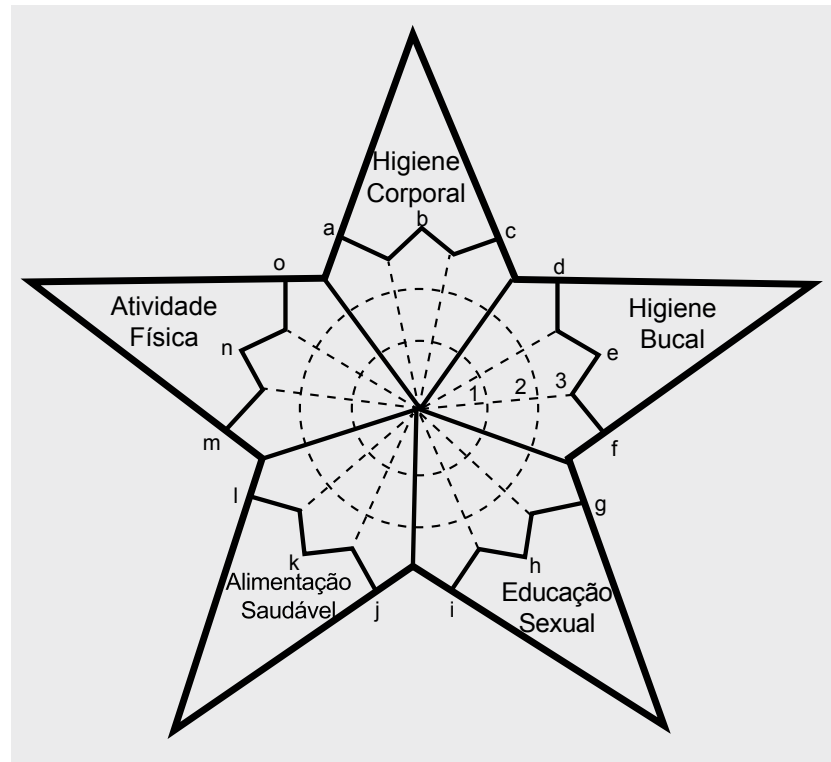

Figura 1 - Pentáculo do bem-estar. Santiago, RS, 2015. Fonte: Adaptado ${ }^{(12)}$

Considerando as respostas dos 15 itens, a participante passa então a colorir o PBE da seguinte forma: deixar em branco se marcou 1 para o item, preencher do centro até o primeiro círculo se marcou 2, preencher do centro até o segundo círculo se marcou 3, e preencher do centro até o terceiro círculo se marcou 4. O preenchimento da estrela acontece sempre do ponto mais interno para o ponto mais externo, e o uso de cores diferentes para colorir possibilita identificar qual é o item mais presente no estilo de vida da participante ${ }^{(12)}$.

A terceira etapa compreendeu a elaboração e a aplicação de oficinas pedagógicas, com as temáticas anteriormente citadas, de forma lúdica e incitando a educação em saúde em lares de adoção. As mesmas continham informações acerca da contaminação por microorganismos. da importância da escovação dentária; do aproveitamento integral dos alimentos; e da prática de exercícios físicos. As atividades foram desenvolvidas sempre no final da tarde, período esse em que todas as residentes encontravam-se no lar.

Cabe destacar que os dados da pesquisa foram obtidos a partir da análise das respostas apresentadas pelas residentes no PBE.

Respeitaram-se os aspectos éticos durante o desenvolvimento da presente pesquisa, a qual foi submetida à avaliação do Comitê de Ética em Pesquisa da Universidade Regional Integrada do Alto Uruguai e Missões - Campus Santiago e foi executada somente após a aprovação, conforme a Resolução 466/12, do Conselho Nacional de Saúde (Parecer: 1.212.676).

\section{RESULTADOS}

Participaram do estudo 9 residentes do sexo feminino, com idade compreendida entre 06 a 17 anos. Todas frequentam a escola pública, e apenas uma delas está no ensino médio, as demais estão no ensino fundamental. Todas as residentes, bem como os coordenadores da instituição, foram participativas e receptivas desde o primeiro encontro, atitude que facilitou o entrosamento e a realização das atividades propostas.

Com base na análise dos resultados, ressaltam-se alguns pontos como, na temática higiene corporal, em que todas as participantes têm o hábito de higienizar as mãos antes das refeições.

Em relação à temática higiene bucal, quando questionadas sobre o uso do fio dental, 5 residentes $(55,5 \%)$ responderam não ter o hábito de usá-lo diariamente. Outra questão que vale ser ressaltada é a frequência de visitas ao dentista, em que apenas 2 residentes $(22,2 \%)$ afirmaram se consultar periodicamente com esse profissional.

Outro ponto importante se remete ao tema educação sexual, pois, quando questionadas sobre o conhecimento de métodos contraceptivos, incluindo o preservativo, todas as $9(100 \%)$ residentes responderam que conhecem ou já mantiveram contato com algum método.

A respeito do tema alimentação saudável, quanto à frequência de consumo de doces, $6(66,6 \%)$ residentes responderam que fazem parte do seu dia-a-dia, enquanto a mesma porcentagem afirmou que "pouco ou raramente" costumam ingerir refrigerante. Por último, 8 residentes $(88,8 \%)$ afirmaram que possuem uma alimentação variada (proteínas, carboidratos, gorduras, fibras, cálcio e outro minerais). 
Quanto ao tema atividade física, destaca-se que 4 residentes (44,4\%) afirmaram incluir a prática de exercícios no seu lazer.

Analisando individualmente os PBE, é possível observar que as participantes da atividade apresentaram o nível positivo (de 31 a 45 pontos) para os critérios analisados.

A partir dessa análise se elaboraram as oficinas pedagógicas teórico-práticas (quadro II), as quais foram realizadas durante cinco encontros com tempo de duração máximo de 2 horas.

Quadro II - Descrição das oficinas pedagógicas teórico/práticas. Santiago, RS, 2015.

\begin{tabular}{|c|c|c|}
\hline Oficinas Teórico/Práticas & Objetivo & Estratégia Utilizada \\
\hline Higiene Corporal & $\begin{array}{l}\text { Estimular os bons hábitos } \\
\text { de higiene, visando uma } \\
\text { melhor qualidade de vida } \\
\text { bem como sensibilizar } \\
\text { para escolhas saudáveis, } \\
\text { conscientes e responsáveis } \\
\text { em relação à saúde e bem- } \\
\text { estar }\end{array}$ & $\begin{array}{l}\text { Realizou-se a abordagem de alguns conceitos básicos de } \\
\text { higiene, por meio de PowerPoint. Após, desenvolveu-se } \\
\text { a dinâmica intitulada "Aquele aperto de mão": modelo de } \\
\text { atividade didática sobre imunização. As residentes foram } \\
\text { divididas em dois grupos: o grupo contaminador e o grupo } \\
\text { infectado. O grupo contaminador borrifa álcool com tinta } \\
\text { do marca-texto nas mãos e, posteriormente, dá um "aperto } \\
\text { de mão" no grupo que será infectado, onde a finalidade da } \\
\text { atividade foi demonstrar através do lúdico que o líquido } \\
\text { borrifado pelo grupo contaminador poderia representar } \\
\text { um espirro de uma pessoa infectada por um antígeno e, na } \\
\text { ausência de higienização necessária, o mesmo se dissipa entre } \\
\text { os indivíduos do grupo infectado por um simples aperto de } \\
\text { mão. }\end{array}$ \\
\hline Higiene Bucal & $\begin{array}{l}\text { Sensibilizar sobre a } \\
\text { importância de manter } \\
\text { bons hábitos de higiene } \\
\text { bucal }\end{array}$ & $\begin{array}{l}\text { Inicialmente, foi realizada uma conversa informal para } \\
\text { verificar as concepções das residentes sobre o tema abordado. } \\
\text { Na sequência, foi iniciada a explanação da temática por meio } \\
\text { de PowerPoint. Para finalizar, foi preparado um creme dental } \\
\text { caseiro com um produto natural que não oferece riscos à saúde } \\
\text { dos dentes através da mistura: bicarbonato, sal, glicerina, } \\
\text { menta/hortelã na quantidade que desejar. }\end{array}$ \\
\hline Educação Sexual & $\begin{array}{l}\text { Conhecer os diferentes } \\
\text { métodos contraceptivos } \\
\text { existentes. Refletir } \\
\text { sobre a importância da } \\
\text { prevenção da gravidez e } \\
\text { das doenças sexualmente } \\
\text { transmissíveis }\end{array}$ & $\begin{array}{l}\text { As residentes foram instigadas a responder alguns } \\
\text { questionamentos por meio de imagens relacionadas ao tema: } \\
\text { Vocês conhecem algum método exposto na imagem? Qual } \\
\text { deles é eficaz contra doenças sexualmente transmissível e } \\
\text { gravidez indesejada? Após, realizou-se a apresentação do } \\
\text { tema por meio de PowerPoint. Para finalizar, foi proposto } \\
\text { um bingo às residentes visando a fixação das informações de } \\
\text { forma lúdica e interativa. }\end{array}$ \\
\hline Alimentação Saudável & $\begin{array}{l}\text { Proporcionar informações } \\
\text { sobre hábitos alimentares } \\
\text { saudáveis, estimular uma } \\
\text { alimentação saudável }\end{array}$ & $\begin{array}{l}\text { Realizou-se uma conversa informal para verificar concepções } \\
\text { das residentes acerca do tema, logo o mesmo foi abordado de } \\
\text { forma expositiva com o auxílio do PowerPoint. Finalizou-se } \\
\text { a atividade com a oficina, onde ocorreu o preparo de um suco } \\
\text { Detox bem como um bolo feito com o reaproveitamento de } \\
\text { cascas de banana. }\end{array}$ \\
\hline Atividade Física & $\begin{array}{l}\text { Valorizar a atividade física } \\
\text { e o exercício como fatores } \\
\text { que contribuem para a } \\
\text { saúde e a qualidade de } \\
\text { vida }\end{array}$ & $\begin{array}{l}\text { Convidaram-se estudantes do curso de Educação Física para } \\
\text { proporcionar às residentes informações referentes à atividade } \\
\text { física e sua importância para uma vida mais saudável. } \\
\text { O primeiro momento foi uma conversa informal sobre a } \\
\text { importância da prática de atividades físicas o os benefícios } \\
\text { que a mesma pode trazer para saúde. Após, foi realizado } \\
\text { um aquecimento inicial com exercícios físicos seguido de } \\
\text { brincadeiras capazes de estimular essa prática. }\end{array}$ \\
\hline
\end{tabular}




\section{DISCUSSÃO}

Os resultados obtidos no presente estudo possibilitam a discussão de alguns pontos importantes em relação ao estilo de vida das residentes do lar de adoção. De acordo com os dados descritos pelo PBE, é possível perceber as concepções significativas das residentes sobre os temas abordados. Mesmo em condições de desamparo ou marginalização social, sem o cuidado parental convencional, o grupo como um todo e individualmente possui conhecimento acerca dos temas abordados, sendo perceptível, conforme pontuação apresentada no teste, que todas possuem um estilo de vida positivo. Esses dados validam a aplicação dessa metodologia como ferramenta de avaliação do estilo de vida de uma amostra.

Outros estudos fazem uso do PBE como ferramenta investigativa da qualidade de vida de diversas populações. Em pesquisa $^{(14)}$ realizada com 43 acadêmicos, concluintes em Educação Física, do Centro Universitário do Leste de Minas Gerais (Unileste-MG), a ferramenta foi usada para analisar variáveis do estilo de vida (hábitos alimentares, hábitos de atividade física e ocupação do tempo livre). Os dados da pesquisa mostraram que o perfil do estilo de vida do grupo foi positivo, deixando a desejar no componente atividade física. Esperava-se de tal componente um resultado positivo, pois eram acadêmicos do último período de Educação Física e que já possuíam um conhecimento teórico e prático referente ao tema.

Nesse mesmo intuito, o presente estudo avaliou o estilo de vida das residentes e obteve resultados positivos quanto à prática de atividades físicas, uma vez que apresentaram um bom nível de conhecimento sobre o tema. Cabe enfatizar que as condições que enfrentam não as impede de praticarem exercícios físicos, bem como ter informações teóricas e práticas a respeito do tema, ao contrário dos acadêmicos que têm um grande suporte teórico-prático e deixam a desejar.

Outros estudos também retratam a aplicação da técnica no contexto de análise do estilo de vida da população idosa, de adolescentes no ensino médio e das percepções de trabalhadores quanto ao ambiente e às condições de trabalho onde se encontram ${ }^{(15-17)}$.

Embora com índice positivo para estilo de vida, é importante reafirmar ou demonstrar atitudes e/ou práticas que promovam a saúde. As temáticas escolhidas para as oficinas estão entre os assuntos mais relevantes e explorados por pesquisas de educação em saúde, permitindo a transversalidade e o ensino coerente à alfabetização científica ${ }^{(18-24)}$.

Em um estudo ${ }^{(18)}$ realizado com estudantes de Medicina e Enfermagem para avaliar as percepções sobre os cuidados de higiene corporal de residentes de uma área periférica de Marília, São Paulo, usando o método de análise e oficinas educativas, observou-se que houve intensa interação entre os participantes, com troca de informações e afetos, o que leva a pensar que atividades dessa natureza constituem uma possibilidade de se avançar na construção de novos modelos de ensino e de cuidado.

Do mesmo modo, na análise dos dados da presente pesquisa, foi possível observar que as participantes demonstraram ter um nível positivo de conhecimento sobre os principais cuidados de higiene, bem como demonstraram envolvimento na atividade. Dessa forma, é possível reafirmar que, para obter mudanças na forma de agir, é preciso ultrapassar os limites da simples transmissão da informação e avançar para a efetiva participação e envolvimento criativo dos atores.

A temática higiene bucal foi explorada em uma pesquisa ${ }^{(19)}$ realizada com 101 indivíduos, com idades entre 12 e 16 anos, pertencentes a três instituições públicas que atendem adolescentes de diferentes inserções sociais, no município de Bauru, São Paulo. O intuito foi avaliar o impacto de métodos educativos participativos na hora de abordar a temática. A análise dos dados permitiu concluir que programas educativos em saúde bucal que aplicam metodologias participativas, como as oficinas pedagógicas, têm fundamental importância na mudança de hábitos de higiene bucal em adolescentes, independentemente de sua inserção social.

Aspectos referentes à alimentação saudável foram abordados em uma pesquisa ${ }^{(20)}$ com o propósito de oferecer oficinas para ensinar, de maneira didática e prazerosa, o preparo adequado de alimentos, bem como avaliar essa ação como estratégia para a promoção de práticas alimentares saudáveis. Participaram do estudo adultos e idosos, de ambos os sexos, com diagnóstico de uma ou mais doenças e agravos não transmissíveis, especificamente obesidade, diabetes e hipertensão. Os resultados apontaram para a assimilação e para a aplicação do conhecimento no cotidiano, além de uma forte intenção dos participantes em mudar algumas atitudes no preparo e no consumo de alimentos ${ }^{(20)}$.

Essas constatações podem reforçar ainda mais o fato de que os métodos tradicionais de intervenção e ensino devem ceder espaço para ações mais dinâmicas e interativas. Durante o desenvolvimento da oficina sobre a temática alimentação saudável, as residentes da atual pesquisa apresentaram-se entusiasmadas e participativas no preparo dos alimentos, fato positivo, uma vez que atividades práticas facilitam a aprendizagem, bem como, estimulam as mudanças de comportamentos.

$\mathrm{O}$ tema atividade física foi abordado em uma pesquisa ${ }^{(21)}$ com o intuito de investigar a relação entre participação esportiva e a qualidade de vida de adolescentes de uma escola pública do município de Araras, São Paulo. De acordo com os resultados, o esporte durante a adolescência reafirma-se enquanto elemento facilitador na ação, voltado à busca da melhoria da qualidade de vida. $\mathrm{O}$ atual estudo também desenvolveu atividade que estimulou a prática esportiva, bem como, auxiliou as residentes na otimização do espaço do abrigo, uma vez que encontrava-se em desuso.

Por último, a temática relacionada à educação sexual tem sido foco de inúmeros estudos, com ampla diversidade de enfoques teóricos e práticos ${ }^{(22-24)}$. Um estudo ${ }^{(25)}$ explorou o tema com o objetivo de abordar o uso de oficinas pedagógicas como estratégia de educação em saúde. A pesquisa foi realizada em uma escola pública municipal na cidade de Fortaleza, Ceará, por 
meio de quatro oficinas educativas com 30 adolescentes. As oficinas tiveram o intuito de promover a reflexão/ação junto aos participantes sobre os eixos: sexualidade, gênero, DST/AIDS e métodos contraceptivos. Os resultados evidenciaram que o uso de oficinas pedagógicas pode despertar um repensar entre os adolescentes sobre a vivência da saúde sexual e reprodutiva a partir das vulnerabilidades percebidas ${ }^{(25)}$.

Diante do exposto, e como já mencionado, o tema educação sexual está presente em diversos estudos, assim como na presente pesquisa. A partir da análise, constatou-se que esse foi o tema que mais instigou as residentes. Tal fato pode estar associado com a fase da adolescência, pois é uma fase de grandes transformações nos âmbitos físico, hormonal, psicológico e social, gerando grande curiosidade por parte das adolescentes ${ }^{(26-28)}$.

Em contrapartida, vive-se atualmente uma banalização do sexo por meio da erotização, que está presente nas residências por meio da televisão, dos jornais, do rádio, das revistas e principalmente da internet. Além disso, os adolescentes estão iniciando a vida sexual cada vez mais cedo, geralmente de forma desprotegida, o que resulta, em muitas ocasiões, em gravidez de garotas recém-saídas da infância, bem como doenças sexualmente transmissíveis ${ }^{(29,30)}$.

Nesse sentido, ao longo dessa discussão e perante a situação em que essas adolescentes se encontram, torna-se fundamental a busca por novas técnicas de ensino. É por meio de novas maneiras de agir, de argumentar e de ousar que será possível contribuir para que as residentes tenham maiores possibilidades de se tornarem cidadãs capazes de viver a vida de forma saudável, aproveitando os seus prazeres de forma responsável e consciente. Com isso, o uso de oficinas pedagógicas pode contribuir de forma significativa para a promoção da saúde nos lares de adoção, pois permitem o estabelecimento de um espaço de reflexão e de compartilhamento de saberes de forma envolvente e lúdica.

Contudo, ainda existem pouquíssimos trabalhos relacionados a esses espaços, sendo um ambiente extremamente prazeroso e gratificante para o desenvolvimento de pesquisas, necessitando ainda ser explorado. Assim, torna-se necessária a aplicação de novos trabalhos direcionados à promoção da saúde nesses espaços.

\section{CONCLUSÃO}

O presente estudo, realizado com as residentes de um lar de adoção, apontou uma percepção positiva de estilo de vida de acordo com os parâmetros do instrumento utilizado. $\mathrm{O}$ uso de oficinas pedagógicas como ferramenta mediadora do processo de educação em saúde foi primordial para o desenvolvimento desse estudo, pois permitiram que as residentes participassem ativamente da construção de um processo educativo e possibilitaram reflexões sobre atitudes e comportamentos favoráveis ao bem-estar do indivíduo.

\section{AGRADECIMENTOS}

Ao Lar das Meninas e a todos os seus integrantes que colaboraram para a realização deste trabalho.

\section{REFERÊNCIAS}

1. Campos DO, Rodrigues JF Neto. Qualidade de vida: um instrumento para promoção de saúde. Rev Baiana Saúde Pública. 2008;32(2):232-40.

2. Maeyama MA, Jasper CH, Nilson LGD, Dolny LL, Cutolo LRA. Promoção da saúde como tecnologia para transformação social. Rev Bras Tecnologias Sociais. 2015;2(2):129-43.

3. Souza AR, Moraes LMP, Barros MGT, Vieira NFC, Braga VAB. Estresse e ações de educação em saúde: contexto da promoção da saúde mental no trabalho. Rev RENE. 2007;8(2):26-34.

4. Morais NA, Morais CA, Reis S, Koller SH. Promoção de saúde e adolescência: um exemplo de intervenção com adolescentes em situação de rua. Psicol Soc. 2010;22(3):507-18.

5. Casarin MR, Picolli JCE. Educação em saúde para prevenção do câncer de colo do útero em mulheres do Município de Santo Ângelo/RS. Ciênc Saúde Coletiva. 2011;16(9):3925-32.

6. Ameida JRS, Oliveira NC, Mourae RF, Saboia VPA, Mota MV, Pinho LGM. Oficinas de promoção de saúde com adolescentes: relato de experiência. Rev RENE. 2011;12(n. esp.):1052-18.

7. Guimarães G, Aerts D, Câmara SG. A escola promotora da saúde e o desenvolvimento de habilidades sociais. Diaphora. 2014;12(2):88-95.

8. Conselho Nacional de Justiça (BR). Cadastro Nacional de Crianças e Adolescentes Acolhidos [acesso em 2017 Jan 01]. Disponível em: http://www.cnj.jus.br/cnca/publico

9. Oliveira APG, Milnitisky-Sapiro C. Abrigos para adolescentes: função de lar transitório? In: II Simpósio Internacional do Adolescente; Maio; São Paulo; 2005. 
10. Ceccim RB, Feuerwerker LCM. O quadrilátero da formação para a área da saúde: ensino, gestão, atenção e controle social. Physis (Rio de J). 2004;14(1):41-65.

11. Nahas MV. Atividade física, saúde e qualidade de vida. Londrina: Midiograf; 2013.

12. Vicente ANC, Picolo AFO, Gomes CM, Viebig RF. Aplicabilidade do Pentáculo do Bem estar como ferramenta para nutricionistas. EFDeportes.com [Internet]. 2009 [acesso em 2016 jul 20];13(129). Disponível em: http://www.efdeportes. com/efd129/aplicabilidade-do-pentaculo-do-bem-estar.htm

13. Dias MSA, Parente JRF, Vasconcelos MIO, Dias FAC. Intersetorialidade e estratégia Saúde da Família: tudo ou quase nada a ver? Ciênc Saúde Coletiva. 2014;19(11):4371-82.

14. Santos, GLA, Venâncio SE. Perfil do estilo de vida de acadêmicos concluintes em educação física do Centro Universitário do Leste de Minas Gerais UNILESTE -MG. Movimentum. 2006;1:1-18.

15. Valle PS, Borgatto AF. Reprodutibilidade de uma escala para avaliar a percepção dos trabalhadores quanto ao ambiente e às condições de trabalho. Rev Bras Saúde Ocup. 2009;34(120):179-83.

16. Silva LWS, Santos RG, Squarcini CFR, Souza AL, Azevedo MP, Barbosa FNM. Perfil do estilo de vida e autoestima da pessoa idosa: Perspectivas de um Programa de Treinamento Físico. Rev Faculdade Ciências Humanas Saúde. 2011;14 (3):145-66.

17. Nahas MV, De Barros MVG, Francalacci V. O pentáculo do bem estar base conceitual para avaliação do estilo de vida de indivíduos ou grupos. Rev Bras Ativ Fís Saúde. 2000;5(2):48-59.

18. Roma E, Silva JA, Magolbo NG, Aquino RF, Marins JS, Moravcik MYAD. Analisando a percepção de uma população sobre higiene a partir de um jogo educativo. Rev Pesquisa Saúde. 2011;12(1):41-6.

19. Tomita NE, Pernambuco AR, Lauris JRP, Lopes ES. Educação em saúde bucal para adolescentes: uso de métodos participativos. Rev Fac Odontol Bauru. 2001;9(1/2):63-9.

20. Capobiango M, Ribeiro RC, Silva AR, Assunção IB, Porto PBV. Oficinas culinárias como estratégia para a promoção de práticas alimentares saudáveis. Percurso Acadêmico. 2014;4 (8):253-67.

21. Gáspari JC, Schwartz GM. Adolescência, esporte e qualidade de vida. Motriz Rev Educ Fís (Impr). 2001;7(2):107-13.

22. Soares SM, Amaral DA, Silva LB, Silva PAB. Oficinas sobre sexualidade na adolescência: revelando vozes, desvelando olhares de estudantes do ensino médio. Esc Anna Nery Rev Enferm. 2008;12(3):485-91.

23. Cajaiba RL. Percepção sobre sexualidade pelos adolescentes antes e após a participação em oficinas pedagógicas. Rev Elect Enseñanza Ciencias. 2013;12(2):234-42.

24. Parker D, Mcgray R. Tensions between teaching sexuality education and neoliberal policy reform in quebec's professional competencies for beginning teachers. McGill J Educ. 2015;50 (1):145-59.

25. Gubert FDA, Santos ACID, Aragão KA, Pereira DCR, Vieira NFC, Pinheiro PNDC. Tecnologias educativas no contexto escolar: estratégia de educação em saúde em escola pública de Fortaleza - CE. Rev Eletrônica Enferm. 2009;11(1):165-72.

26. Fuzzella L, Fedescob HN, Alexander CSC, Fortenberryd JD, Shieldsa CG. "I just think that doctors need to ask more questions": sexual minority and majority adolescents' experiences talking about sexuality with healthcare providers. Patient Educ Couns. 2016;99(9):1467-72.

27. Viola DTD, Vorcaro AMR. O problema do saber na adolescência e o real da puberdade. Psicol USP. 2015;26(1):62-70.

28. Vigil P, Del rio JP, Carrera BR, Aranguiz FC, Rioseco H, Cortés ME. Influence of sex steroid hormones on the adolescent brain and behavior: An update. Linacre Q. 2016;83(3):308-29.

29. Fonseca ADD, Gomes VLDO, Teieira KC. Percepção de adolescentes sobre uma ação educativa em orientação sexual realizada por acadêmicos (as) de enfermagem. Esc Anna Nery Rev Enferm. 2010;14(2):330-7.

30. Rossi E, Poulin F, Boislard MA. Trajectories of annual number of sexual partners from adolescence to emerging adulthood: individual and family predictors. J Youth Adolesc. 2016;24:1-4.

\section{Endereço para correspondência:}

Cintia Soares Guerin

Avenida Batista Bonoto Sobrinho, 733

Campus Santiago

CEP: 97700-000 - Santiago - Rio Grande do Sul - Brasil

E-mail: cintiaguerin@hotmail.com 